Pacific Journal of Mathematics

TRANSLATION-INVARIANT FUNCTION ALGEBRAS ON 


\title{
TRANSLATION-INVARIANT FUNCTION ALGEBRAS ON COMPACT GROUPS
}

\author{
Joseph A. WoLF
}

\begin{abstract}
Let $X$ be a compact group. $(5(X)$ denotes the Banach algebra (point multiplication, sup norm) of continuous complexvalued functions on $X . A$ is any closed subalgebra of $\sqrt{\varsigma}(X)$ which is stable under right and left translations and contains the constants. It is shown, by means of the Peter-Weyl Theorem and some multilinear algebra, that the condition $(*)$ every representation of degree 1 of $X$ has finite image is necessary and sufficient that every possible $A$ be self-adjoint. If $X$ is connected, then $\left(^{*}\right)$ means that $X$ is a projective limit of semisimple Lie groups; if $X$ is a Lie group, then (*) means that $X$ is semisimple. The Stone-Weierstrass Theorem then gives a quick classification of all possible algebras $A$ on an arbitrary connected semisimple Lie group $X$.
\end{abstract}

In an earlier paper we characterized the compact groups on which every closed translation-invariant ${ }^{(1)}$ function space is self adjoint [1, Theorem 4.1]. An application of the Stone-Weierstrass Theorem [1, § 7] resulted in a classification of the closed translation-invariant function algebras on the connected Lie groups which satisfied the conditions of the characterization. Those Lie groups are the compact connected semisimple Lie groups with no simple component locally isomorphic to $S U(n)(n>2)$, to $S O(4 n+2)$, nor to $E_{6}$.

In this paper we give a direct characterization of the compact groups on which every closed translation-invariant function algebra is self adjoint. For compact connected Lie groups, the characterization is that the group be semisimple. Many of these groups have closed translation-invariant function spaces which are not self adjoint. Finally we classify the closed translation-invariant function algebras on compact simple simply connected Lie groups, as an example of a general enumeration procedure.

2. Notation. Let $X$ be a compact group. $D$ denotes the set of all (equivalence classes of) irreducible finite dimensional representations of $X$. Let $\beta \in D$; then $W^{\beta}$ denotes the representation space, and $\beta^{*}$ is the representation of $X$ on the dual space of $W^{\beta}$ induced by $\beta$. $\beta^{*}$ is called the contragredient of $\beta . \quad E^{\beta}=W^{\beta} \otimes W^{\beta^{*}}$ is identified with the space of matrix functions (coefficients) of $\beta$. Let $C(X)$ denote the

Received April 20, 1964. Partially supported by National Science Foundation Grant GP-812.

(1) Stable under both right and left translations. 
set of continuous complex-valued functions on $X$ with sup norm and point multiplication. Then $C(X)$ is a commutative Banach algebra. The Peter-Weyl Theorem says that every element of $C(X)$ has a Fourier development along $\left\{E^{\beta}\right\}_{\beta \in D}$, i.e.,

$$
C(X) \sim \sum_{\beta \in D} E^{\beta}
$$

The translation group $T(X)$ of $X$ is the group consisting of all transformations

$$
(u, v): x \rightarrow u x v^{-1}, \quad u, v, x \in X,
$$

of $X$. A subset $S \subset C(X)$ is called translation-invariant if $T(X) \cdot S=$ $S$. The closed translation-invariant subspaces of $C(X)$ are just the subspaces spanned by sets of the form $\left\{E^{\beta}\right\}_{\beta \in F}$ with $F \subset D$.

If $X$ is a compact connected Lie group endowed with a Riemannian metric invariant under both right and left translations, then $X$ is a Riemannian symmetric space and $T(X)$ is the largest connected group of isometries.

A subspace of $C(X)$ is called self-adjoint if it is closed under complex conjugation of functional values.

Let $\alpha$ and $\beta$ be representations of a group $Y$, with finite dimensional representation spaces $V$ and $W$. Then $V \otimes W$ denotes the tensor product. If $\left\{v_{i}\right\}$ is a basis of $V$ and $\left\{w_{j}\right\}$ is a basis of $W$, then $\left\{v_{i} \otimes w_{j}\right\}$ is a basis of $V \otimes W$. We have matrix functions defined by

$$
\begin{aligned}
& \alpha(y) \cdot v_{i}=\sum_{u} \alpha_{i u}(y) \cdot v_{u} \\
& \beta(y) \cdot w_{i}=\sum_{t} \beta_{j t}(y) \cdot w_{t} .
\end{aligned}
$$

Now let $\alpha \otimes \beta$ be the representation of $Y$ on $V \otimes W$ given by

$$
(\alpha \otimes \beta)(y) \cdot v \otimes w=\{\alpha(y) \cdot v\} \otimes\{\beta(y) \cdot w\} .
$$

$\alpha \otimes \beta$ is given on the basis $\left\{v_{i} \otimes w_{j}\right\}$ by

$$
(\alpha \otimes \beta)(y) \cdot v_{i} \otimes w_{j}=\sum_{u, t} \alpha_{i u}(y) \beta_{j t}(y) v_{u} \otimes w_{t}
$$

Let $\alpha$ be a representation of a group $Y$ on a finite dimensional space $V$. The symmetric group $S_{r}$ on $r$ letters acts on $V \otimes \cdots \otimes V$ ( $r$ times) by permutation of the factors. The alternation $A^{r}(V)$ consists of all elements $p \in V \otimes \cdots \otimes V(r$ times $)$ such that $\sigma(p)=(\operatorname{sign} \sigma) p$ for all $\sigma \in S_{r}$. If $\left\{v_{1}, \cdots, v_{n}\right\}$ is a basis of $V$, then $\Lambda^{r}(V)$ has a basis consisting of all $v_{i_{1}} \wedge v_{i_{2}} \wedge \cdots \wedge v_{i_{r}}$ where $1 \leqq i_{1}<i_{2}<\cdots<i_{r} \leqq n$ and

$$
v_{i_{1}} \wedge \cdots \wedge v_{i_{r}}=\sum_{\sigma \in S_{r}}(\operatorname{sign} \sigma) v_{i_{\sigma(1)}} \otimes \cdots \otimes v_{i_{\sigma(r)}}
$$


For example, if $r=2$ then $v_{1} \wedge v_{2}=v_{1} \otimes v_{2}-v_{2} \otimes v_{1} ; v_{1} \wedge \cdots \wedge v_{n}$ spans $\Lambda^{n}(V)$. The action of $\alpha \otimes \cdots \otimes \alpha$ on $V \otimes \cdots \otimes V$ commutes with the action of $S_{r}$. Thus $\alpha \otimes \cdots \otimes \alpha$ ( $r$ times) preserves the subspace $A^{r}(V)$ of $V \otimes \cdots \otimes V$. The representation on that subspace, the so-called $r$ th alternation of $\alpha$, is denoted $\Lambda^{r}(\alpha)$. If $\alpha(y) \cdot v_{i}=$ $\sum_{j} \alpha_{i j}(y) \cdot v_{j}$, then one checks that $\Lambda^{r}(\alpha y) \cdot v_{(i)}=\sum_{(j)} A_{(i)(j)}(y) \cdot v_{(j)}$ where $v_{(i)}=v_{i_{1}} \wedge \cdots \wedge v_{i_{r}}$ and

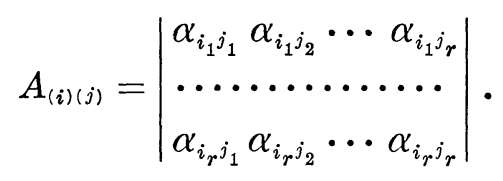

3. The characterization. Our main result is:

3.1. Theorem. Let $X$ be a compact topological group. Then every closed translation-invariant subalgebra of $C(X)$ is self adjoint, if and only if, every representation of degree 1 of $X$ has finite image.

3.2. CoROLlary. Let $X$ be a compact connected group. Then every closed translation-invariant subalgebra of $C(X)$ is self adjoint, if and only if, $X$ is an inverse limit of semisimple Lie groups.

3.3. CoROllary. Let $X$ be a compact connected Lie group. Then every closed translation-invariant subalgebra of $C(X)$ is self adjoint, if and only if, $X$ is semisimple.

The proof of Theorem 3.1 is based on two simple lemmas.

3.4. Lemma. Let $\lambda$ be a finite dimensional representation of a group $Y$. Then $\lambda^{*}$ is equivalent to the representation

$$
y \rightarrow(\operatorname{det} \cdot \lambda(y))^{-1} \cdot \Lambda^{n}(\lambda(y))
$$

where $n+1$ is the degree of $\lambda$.

Proof. Let $V$ be the representation space and choose a nonzero element $e \in A^{n+1}(V)$. If $g$ is a linear transformation of $V$, then the determinant of $g$ is given by $\Lambda^{n+1}(g) \cdot e=(\operatorname{det} \cdot g) e$. If $w \in A^{n}(V)$, we define a linear functional $f_{w}$ on $V$ by $w \wedge v=f_{w}(v) \cdot e ; w \rightarrow f_{w}$ is an isomorphism of $\Lambda^{n}(V)$ onto the dual space $V^{*}$ of $V$. Let $\nu$ be the representation $y \rightarrow(\operatorname{det} \cdot \lambda(y))^{-1} \cdot \Lambda^{n}(\lambda y)$. Then

$$
\begin{aligned}
\left\{f_{\nu(y) \cdot w}(\lambda(y) \cdot v)\right\} e & =\nu(y) w \wedge \lambda(y) v \\
& =(\operatorname{det} \cdot \lambda(y))^{-1} \cdot A^{n+1}(\lambda y)(w \wedge v) \\
& =w \wedge v=f_{w}(v) \cdot e .
\end{aligned}
$$


Thus the isomorphism $w \rightarrow f_{w}$ induces an equivalence of $\nu$ with $\lambda^{*}$.

3.5. Lemma. Let $\lambda$ and $\mu$ be finite dimensional representations of a group $Y$. Then every matrix function of $\Lambda^{r}(\lambda)$ is a linear combination of products of $r$ matrix functions of $\lambda$, and every matrix function of $\lambda \otimes \mu$ is a linear combination of products of matrix functions of $\lambda$ with matrix functions of $\mu$.

Proof. Obvious from the definitions of $\Lambda^{r}(\lambda)$ and $\lambda \otimes \mu$.

3.6. Proof of Theorem 3.1. Suppose first that $X$ has a representation $\pi$ of degree 1 with infinite image. Then $\pi(X)$ is a circle group consisting of the unimodular complex numbers, and $\pi^{n}: x \rightarrow \pi(x)^{n}$ is another representation of degree 1. Let $A$ be the subalgebra of $C(X)$ spanned by the $E^{\pi^{n}}, n \geqq 0$. Then $A$ is a closed translation-invariant subalgebra of $C(X) ; \pi \in A$ and $\bar{\pi} \notin A$, so $A$ is not self adjoint.

Now suppose that every representation of degree 1 of $X$ has finite image and let $A$ be a closed translation-invariant subalgebra of $C(X)$. Then $A$ is spanned by subspaces $E^{\beta} \subset C(X)$, say for $\beta$ running over a subset $D_{A} \subset D$, and we must prove that $A$ contains the adjoint of $E^{\beta}$ for every $\beta \in D_{A}$. In orthonormal dual bases of the representation spaces, $\overline{\beta(x)}={ }^{t} \beta(x)^{-1}$ for every $x \in X$; thus $E^{\beta^{*}}$ is the adjoint of $E^{\beta}$. Now we need only prove that $\beta \in D_{A}$ implies $\beta^{*} \in D_{A}$.

Let $\alpha \in D_{A}$ and define $\gamma(x)=\operatorname{det} \cdot \alpha(x)$. Then $\gamma=\Lambda_{r}(\alpha)$ where $r$ is the degree of $\alpha$, so $\gamma \in D_{A}$ by Lemma 3.5. $\gamma(X)$ is finite by hypothesis; thus $\gamma(X)$ is cyclic of some finite order $n$, consisting of the numbers $e^{2 \pi i k / n}$. Now $\bar{\gamma}=\gamma^{-1}=\gamma^{*}=\gamma^{n-1} \in A$, so $\gamma^{-1} \in D_{A}$. $\Lambda^{r-1}(\alpha) \in D_{A}$ and $D_{A}$ is closed under $\otimes$, by Lemma 3.5; thus $\gamma^{-1} \otimes \Lambda^{r-1}(\alpha) \in D_{A}$. Lemma 3.4 says $\alpha^{*}=\gamma^{-1} \otimes \Lambda^{r-1}(\alpha)$; thus $\alpha^{*} \in D_{A}$.

3.7. Proof of corollaries. If $X$ is compact connected Lie group, then $X=X^{\prime} \cdot T$ where $X^{\prime}$ is the derived group, $T$ is the identity component of the center of $X$, and $T \cap X^{\prime}$ is finite. $T$ is a product of circle groups, $X^{\prime}$ is semisimple, and the following conditions are equivalent: (i) $X$ is semisimple, (ii) $X=X^{\prime}$, and (iii) $T=\{1\}$.

Let $X$ be semisimple and let $\alpha$ be a representation of degree 1 . $\alpha(X)$ is commutative, so the kernel of $\alpha$ contains $X^{\prime}=X$. Thus $\alpha(X)$ is finite.

Let $X$ be not semisimple. Then $S=T / T \cap X^{\prime}$ is a torus of positive dimension, so there is a nontrivial representation $\beta$ of $S$ of degree 1 . If $\pi: X \rightarrow X / X^{\prime}=S$ is the projection, then $\alpha=\beta \cdot \pi$ is a representation of degree 1 of $X$ and $\alpha(X)=\beta(S)$ is infinite.

Corollary 3.3 is proved. 
Let $X$ be a compact connected group. If $\pi$ is a finite dimensional representation, we have the Lie group $\pi(X) ;\{\pi(X)\}$ is an inverse system of compact Lie groups with $X$ as inverse limit, and $\{\pi(X)\}$ is maximal for this property. A representation $\alpha$ is of degree 1 with infinite image, if and only if $\alpha(X)$ is a circle group. If each $\pi(X)$ is semisimple, then none is a circle group. If some $\pi(X)$, say $\alpha(X)$, is a circle group, then $\beta(X)$ is not semisimple for $\beta>\alpha$, so $X$ cannot be an inverse limit of a subsystem of $\{\pi(X)\}$ which consists of semisimple groups. Corollary 3.2 follows.

4. Enumeration of self adjoint function algebras. The enumeration of closed translation-invariant self adjoint function algebras on a compact group is based on the following combination of the Peter-Weyl Theorem and the Stone-Weierstrass Theorem.

4.1. Theorem. Let $X$ be a compact topological group, let $D$ be the set of equivalence classes of the irreducible representations of $X$, and, given $\pi \in D$, let $E^{\pi}$ denote the space of matrix functions of $\pi$. Then there are one-to-one correspondences between

(i) closed self adjoint translation-invariant subalgebras $A$ of $C(X)$ which contain the constants,

(ii) subsets $F \subset D$ with the properties (a) $1_{x} \in F$, (b) if $\beta \in F$ then $\beta^{*} \in F$ and (c) if $\alpha, \beta \in F$ then $F$ contains every irreducible summand of $\alpha \otimes \beta$, and

(iii) closed normal subgroups $\Gamma \subset X$.

The correspondences are given by

$$
A \sim \sum_{\beta \in F} E^{\beta} \quad \text { and } \quad A=C(X / \Gamma)
$$

where $C(X / \Gamma) \subset C(X)$ by defining $f(x)=f(x \Gamma)$ for $f \in C(X / \Gamma)$.

4.2. COROLLARY. If $X$ is a compact group for which every representation of degree 1 has finite image, then the closed translationinvariant subalgebras of $C(X)$ which contain the constants, are just the algebras $C(Y)$ where $Y$ is a quotient group of $X$.

Corollary 4.2 is an immediate consequence of Theorems 3.1 and 4.1.

4.3. Proof of Theorem 4.1. Let $F \subset D$ and define $A_{F}$ to be the subspace of $C(X)$ spanned by $\left\{E^{\beta}\right\}_{\beta \in F}$. Then $A_{F}$ is a closed translationinvariant subspace of $C(X)$. Condition (iia) says that $A_{F}$ contains the constants, condition (iib) says that $A_{F}$ is self adjoint, and condition (iic) says that $A_{F}$ is an algebra. Thus $A \sim \sum_{\beta \in F} E^{\beta}$ gives a one-to-one correspondence between the classes (i) and (ii). 
Let $F \subset D$ satisfy conditions (iia, b, c), and define $\Gamma_{F}$ to be the intersection of the kernels of the elements of $F$. Then $\Gamma_{F}$ is a closed normal subgroup of $X$. Let $p: X \rightarrow X / \Gamma_{F}$ be the projection and define $D_{F}$ to be the set of irreducible representations of $X / \Gamma_{F}$. Then $D_{F} \subset D$ under $\sigma \rightarrow \sigma \cdot p$. Now $A_{F^{\prime}} \subset C\left(X / \Gamma_{F}\right)$ under $f\left(x_{F}\right)=f(x)$, and $A_{F}=$ $C\left(X / \Gamma_{F}\right)$ by the Stone-Weierstrass Theorem. It follows that $D_{F}=F$. Thus $\Gamma \leftrightarrow\{\beta \in D: \Gamma \subset$ Kernel. $\beta\}$ is a one-to-one correspondence between the classes (iii) and (ii), and the composition of this with our other correspondence is given by $\Gamma \leftrightarrow C(X / \Gamma)$.

4.4. Application to compact simply connected Lie groups. Let $X$ be a compact simply connected Lie group. Then $X=X_{1} \times \cdots \times X_{r}$ where the $X_{i}$ are compact simply connected simple Lie groups. By Corollary 3.3 and Theorem 4.1, the closed translation-invariant subalgebras of $C(X)$ containing the constants are enumerated by the enumeration of the closed normal subgroups $\Gamma \subset X$. $X$ has center $Z=Z_{1} \times \cdots \times Z_{r}$ where $Z_{i}$ is the center of $X_{i}$, and the closed normal subgroups of $X$ are just the groups $\Gamma=X^{\prime} \cdot Z^{\prime}$ where

(i) $X^{\prime}$ is a product of zero or more of the groups $X_{i}$ and

(ii) $Z^{\prime}$ is a subgroup of $Z$.

Thus our enumeration problem is reduced to a knowledge of the centers of the groups $X_{i}$. This information is known; it is summarized in the following table for the convenience of the reader. Here $Z_{(m)}$ denotes the cyclic group of order $m$ and the groups listed are all the compact simple simply connected Lie groups.

For example, the number of closed translation-invariant subalgebras

TABLE 1

\begin{tabular}{|c|c|c|}
\hline group & description & center \\
\hline $\begin{array}{l}S U(n) \\
(n>1)\end{array}$ & $\begin{array}{l}\text { special unitary group } \\
\text { in } n \text { complex variables }\end{array}$ & $Z_{(n)}$ \\
\hline $\begin{array}{l}\text { Spin }(n) \\
(2<n \neq 4)\end{array}$ & $\begin{array}{l}\text { two sheeted covering } \\
\text { group of the rotation } \\
\text { group in } n \text { real variables }\end{array}$ & $\begin{array}{l}Z_{(2)}(n \text { odd }), \\
Z_{(2)} \times Z_{(2)}(n=4 k), \\
Z_{(4)}(n=4 k+2)\end{array}$ \\
\hline$S p(n)$ & $\begin{array}{l}\text { unitary group in } n \\
\text { quaternion variables }\end{array}$ & $Z_{(2)}$ \\
\hline$G_{2}$ & $\begin{array}{l}\text { automorphism group of } \\
\text { the Cayley algebra }\end{array}$ & $\{1\}$ \\
\hline$F_{4}$ & $\begin{array}{l}\text { elliptic group of the } \\
\text { Cayley projective plane }\end{array}$ & $\{1\}$ \\
\hline$E_{6}$ & $\cdot \cdot \cdot \cdot$ & $Z_{(3)}$ \\
\hline$E_{7}$ & $\cdots \cdot$ & $Z_{(2)}$ \\
\hline$E_{8}$ & $\cdot \cdot \cdot \cdot$ & $\{1\}$ \\
\hline
\end{tabular}


of $C(X)$ properly containing the constants is one (just $C(X)$ ) for $X=$ $G_{2}, F_{4}$ or $E_{8}$; it is two for $X=\operatorname{Spin}(2 k+1), S p(n), E_{6}$ or $E_{7}$; it is three for $X=\operatorname{Spin}(4 k+2)$ and five for $X=\operatorname{Spin}(4 k)$; for $X=S U(n)$ it is the number of divisors of $n$, counting both 1 and $n$.

\section{REFERENCE}

1. J. A. Wolf, Self adjoint funciion spaces on Riemannian symmetric manifolds, Trans. Amer. Math. Soc. 113 (1964), 299-315.

University of CALIFornia, Berkeley 



\section{PACIFIC JOURNAL OF MATHEMATICS}

\section{EDITORS}

\author{
H. Samelson \\ Stanford University \\ Stanford, California \\ R. M. Blumenthal \\ University of Washington \\ Seattle, Washington 98105
}

\author{
J. Dugundu \\ University of Southern California \\ Los Angeles, California 90007 \\ *Richard Arens \\ University of California \\ Los Angeles, California 90024
}

\section{ASSOCIATE EDITORS}
E. F. BeCKENBACH
B. H. NeUMANN
F. WOLF
K. YosidA

\section{SUPPORTING INSTITUTIONS}

\author{
UNIVERSITY OF BRITISH COLUMBIA \\ CALIFORNIA INSTITUTE OF TECHNOLOGY \\ UNIVERSITY OF CALIFORNIA \\ MONTANA STATE UNIVERSITY \\ UNIVERSITY OF NEVADA \\ NEW MEXICO STATE UNIVERSITY \\ OREGON STATE UNIVERSITY \\ UNIVERSITY OF OREGON \\ OSAKA UNIVERSITY \\ UNIVERSITY OF SOUTHERN CALIFORNIA
}

\author{
STANFORD UNIVERSITY \\ UNIVERSITY OF TOKYO \\ UNIVERSITY OF UTAH \\ WASHINGTON STATE UNIVERSITY \\ UNIVERSITY OF WASHINGTON \\ * * * * \\ AMERICAN MATHEMATICAL SOCIETY \\ CALIFORNIA RESEARCH CORPORATION \\ SPACE TECHNOLOGY LABORATORIES \\ NAVAL ORDNANCE TEST STATION
}

Mathematical papers intended for publication in the Pacific Journal of Mathematics should by typewritten (double spaced). The first paragraph or two must be capable of being used separately as a synopsis of the entire paper. It should not contain references to the bibliography. No separate author's resumé is required. Manuscripts may be sent to any one of the four editors. All other communications to the editors should be addressed to the managing editor, Richard Arens, at the University of California, Los Angeles, California 90024.

50 reprints per author of each article are furnished free of charge; additional copies may be obtained at cost in multiples of 50 .

The Pacific Journal of Mathematics is published quarterly, in March, June, September, and December. Effective with Volume 13 the price per volume (4 numbers) is $\$ 18.00$; single issues, $\$ 5.00$. Special price for current issues to individual faculty members of supporting institutions and to individual members of the American Mathematical Society: $\$ 8.00$ per volume; single issues $\$ 2.50$. Back numbers are available.

Subscriptions, orders for back numbers, and changes of address should be sent to Pacific Journal of Mathematics, 103 Highland Boulevard, Berkeley 8, California.

Printed at Kokusai Bunken Insatsusha (International Academic Printing Co., Ltd.), No. 6, 2-chome, Fujimi-cho, Chiyoda-ku, Tokyo, Japan.

PUBLISHED BY PACIFIC JOURNAL OF MATHEMATICS, A NON-PROFIT CORPORATION

The Supporting Institutions listed above contribute to the cost of publication of this Journal, but they are not owners or publishers and have no responsibility for its content or policies.

* Basil Gordon, Acting Managing Editor until February 1, 1966. 


\section{Pacific Journal of Mathematics}

\section{Vol. 15, No. $3 \quad$ November, 1965}

David R. Arterburn and Robert James Whitley, Projections in the space of

bounded linear operators .................................

Robert McCallum Blumenthal, Joram Lindenstrauss and Robert Ralph Phelps,

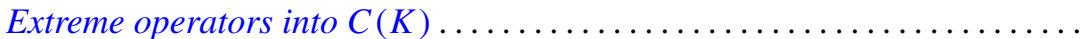

L. Carlitz, A note on multiple exponential sums ................... 757

Joseph A. Cima, A nonnormal Blaschke-quotient .................... 767

Paul Civin and Bertram Yood, Lie and Jordan structures in Banach algebras . . . 775

Luther Elic Claborn, Dedekind domains: Overrings and semi-prime

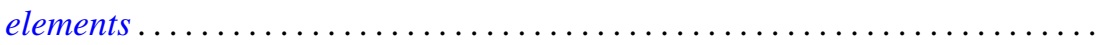

799

Luther Elic Claborn, Note generalizing a result of Samuel's .............. 805

George Bernard Dantzig, E. Eisenberg and Richard Warren Cottle, Symmetric

dual nonlinear programs ................................... 809

Philip J. Davis, Simple quadratures in the complex plane ............... 813

Edward Richard Fadell, On a coincidence theorem of F. B. Fuller ............ 825

Delbert Ray Fulkerson and Oliver Gross, Incidence matrices and interval

graphs ........................................ 835

Larry Charles Grove, Tensor products over $H^{*}$-algebras ..................

Deborah Tepper Haimo, $L^{2}$ expansions in terms of generalized heat polynomials

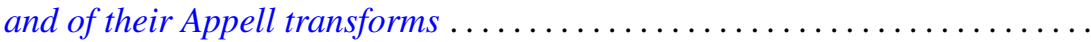

I. Martin (Irving) Isaacs and Donald Steven Passman, A chardcterization of groups in terms of the degrees of their characters ..........

Donald Gordon James, Integral invariants for vectors over local fields........ 905

Fred Krakowski, A remark on the lemma of Gauss ................... 917

Marvin David Marcus and H. Minc, A subdeterminant inequality ........... 921

Kevin Mor McCrimmon, Norms and noncommutative Jordan algebras ........ 925

Donald Earl Myers, Topologies for Laplace transform spaces ............... 957

Olav Njstad, On some classes of nearly open sets .................... 961

Milton Philip Olson, A characterization of conditional probability ........... 971

Barbara Osofsky, A counter-example to a lemma of Skornjakov .............. 985

Sidney Charles Port, Ratio limit theorems for Markov chains ............... 989

George A. Reid, A generalisation of $W^{*}$-algebras ...................... 1019

Robert Wells Ritchie, Classes of recursive functions based on Ackermann's

function ........................................... 1027

Thomas Lawrence Sherman, Properties of solutions of $n$th order linear

differential equations ........................................ 1045

Ernst Snapper, Inflation and deflation for all dimensions . .............. 1061

Kondagunta Sundaresan, On the strict and uniform convexity of certain Banach

spaces............................................. 1083

Frank J. Wagner, Maximal convex filters in a locally convex space .......... 1087

Joseph Albert Wolf, Translation-invariant function algebras on compact 